\title{
Two-photon production of charged pion and kaon pairs
}

J. Dominick, ${ }^{1}$ M. Lambrecht, ${ }^{1}$ S. Sanghera, ${ }^{1}$ V. Shelkov, ${ }^{1}$ T. Skwarnicki, ${ }^{1}$ R. Stroynowski, ${ }^{1}$ I. Volobouev, ${ }^{1}$ G. Wei, ${ }^{1}$ P. Zadorozhny, ${ }^{1}$ M. Artuso, ${ }^{2}$ M. Goldberg, ${ }^{2}$ D. He,${ }^{2}$ N. Horwitz ${ }^{2}$ R. Kennett, ${ }^{2}$ R. Mountain, ${ }^{2}$ G.C. Moneti, ${ }^{2}$ F. Muheim,$^{2}$ Y. Mukhin, ${ }^{2}$ S. Playfer, ${ }^{2}$ Y. Rozen, ${ }^{2}$ S. Stone,${ }^{2}$ M. Thulasidas, ${ }^{2}$ G. Vasseur, ${ }^{2}$ G. Zhu $^{2}{ }^{2}$ J. Bartelt ${ }^{3}$ S.E. Csorna, ${ }^{3}$ Z. Egyed, ${ }^{3}$ V. Jain ${ }^{3}$ K. Kinoshita, ${ }^{4}$ K.W. Edwards, ${ }^{5}$ M. Ogg, ${ }^{5}$ D.I. Britton, ${ }^{6}$ E.R.F. Hyatt, ${ }^{6}$ D.B. MacFarlane, ${ }^{6}$ P.M. Patel, ${ }^{6}$ D.S. Akerib, ${ }^{7}$ B. Barish, ${ }^{7}$ M. Chadha, ${ }^{7}$ S. Chan, ${ }^{7}$ D.F. Cowen, ${ }^{7}$ G. Eigen, ${ }^{7}$ J.S. Miller, ${ }^{7}$ C. O'Grady, ${ }^{7}$ J. Urheim, ${ }^{7}$ A.J. Weinstein, ${ }^{7}$ D. Acosta, ${ }^{8}$ M. Athanas, ${ }^{8}$ G. Masek, ${ }^{8}$ H.P. Paar, ${ }^{8}$ M. Sivertz ${ }^{8}$ J. Gronberg, ${ }^{9}$ R. Kutschke, ${ }^{9}$ S. Menary, ${ }^{9}$ R.J. Morrison, ${ }^{9}$ S. Nakanishi,${ }^{9}$ H.N. Nelson, ${ }^{9}$ T.K. Nelson, ${ }^{9}$ C. Qiao, ${ }^{9}$ J.D. Richman, ${ }^{9}$ A. Ryd,${ }^{9}$ H. Tajima, ${ }^{9}$ D. Sperka,${ }^{9}$ M.S. Witherell, ${ }^{9}$ M. Procario, ${ }^{10}$ R. Balest,${ }^{11}$ K. Cho, ${ }^{11}$ M. Daoudi, ${ }^{11}$ W.T. Ford, ${ }^{11}$ D.R. Johnson, ${ }^{11}$ K. Lingel, ${ }^{11}$ M. Lohner, ${ }^{11}$ P. Rankin, ${ }^{11}$ J.G. Smith, ${ }^{11}$ J.P. Alexander, ${ }^{12}$ C. Bebek, ${ }^{12}$ K. Berkelman, ${ }^{12}$ K. Bloom,${ }^{12}$ T.E. Browder, ${ }^{12, *}$ D.G. Cassel, ${ }^{12}$ H.A. Cho, ${ }^{12}$ D.M. Coffman, ${ }^{12}$ P.S. Drell, ${ }^{12}$ R. Ehrlich, ${ }^{12}$ P. Gaiderev ${ }^{12}$ R.S. Galik, ${ }^{12}$ M. Garcia-Sciveres, ${ }^{12}$ B. Geiser, ${ }^{12}$ B. Gittelman, ${ }^{12}$ S.W. Gray, ${ }^{12}$ D.L. Hartill, ${ }^{12}$ B.K. Heltsley, ${ }^{12}$ C.D. Jones, ${ }^{12}$ S.L. Jones, ${ }^{12}$ J. Kandaswamy, ${ }^{12}$ N. Katayama, ${ }^{12}$ P.C. Kim ${ }^{12}$ D.L. Kreinick, ${ }^{12}$ G.S. Ludwig, ${ }^{12}$ J. Masui, ${ }^{12}$ J. Mevissen, ${ }^{12}$ N.B. Mistry, ${ }^{12}$ C.R. Ng, ${ }^{12}$ E. Nordberg, ${ }^{12}$ J.R. Patterson, ${ }^{12}$ D. Peterson, ${ }^{12}$ D. Riley, ${ }^{12}$ S. Salman, ${ }^{12}$ M. Sapper, ${ }^{12}$ F. Würthwein, ${ }^{12}$ P. Avery, ${ }^{13}$ A. Freyberger, ${ }^{13}$ J. Rodriguez ${ }^{13}$ R. Stephens, ${ }^{13}$ S. Yang, ${ }^{13}$ J. Yelton, ${ }^{13}$ D. Cinabro, ${ }^{14}$ S. Henderson, ${ }^{14}$ T. Liu, ${ }^{14} \mathrm{M}$. Saulnier, ${ }^{14}$ R. Wilson, ${ }^{14}$ H. Yamamoto, ${ }^{14}$ T. Bergfeld, ${ }^{15}$ B.I. Eisenstein, ${ }^{15}$ G. Gollin, ${ }^{15}$ B. Ong,${ }^{15}$ M. Palmer, ${ }^{15}$ M. Selen, ${ }^{15}$ J. J. Thaler, ${ }^{15}$ A.J. Sadoff, ${ }^{16}$ R. Ammar, ${ }^{17}$ S. Ball, ${ }^{17}$ P. Baringer,${ }^{17}$ A. Bean, ${ }^{17}$ D. Besson, ${ }^{17}$ D. Coppage,${ }^{17}$ N. Copty, ${ }^{17}$ R. Davis,${ }^{17}$ N. Hancock, ${ }^{17}$ M. Kelly, ${ }^{17}$ N. Kwak,${ }^{17}$ H. Lam,${ }^{17}$ Y. Kubota,${ }^{18}$ M. Lattery, ${ }^{18}$ J.K. Nelson, ${ }^{18}$ S. Patton, ${ }^{18}$ D. Perticone ${ }^{18}$ R. Poling, ${ }^{18}$ V. Savinov, ${ }^{18}$ S. Schrenk, ${ }^{18}$ R. Wang, ${ }^{18}$ M.S. Alam, ${ }^{19}$ I.J. Kim, ${ }^{19}$ B. Nemati, ${ }^{19}$ J.J. O'Neill, ${ }^{19}$ H. Severini, ${ }^{19}$ C.R. Sun, ${ }^{19}$ M.M. Zoeller, ${ }^{19}$ G. Crawford,${ }^{20}$ C. M. Daubenmier, ${ }^{20}$ R. Fulton, ${ }^{20}$ D. Fujino, ${ }^{20}$ K.K. Gan, ${ }^{20}$ K. Honscheid, ${ }^{20}$ H. Kagan, ${ }^{20}$ R. Kass, ${ }^{20}$ J. Lee ${ }^{20}$ R. Malchow, ${ }^{20}$ Y. Skovpen, ${ }^{20}+\dagger$ M. Sung, ${ }^{20}$ C. White ${ }^{20}$ F. Butler,${ }^{21}$ X. Fu, ${ }^{21}$ G. Kalbfleisch, ${ }^{21}$ W.R. Ross, ${ }^{21}$ P. Skubic, ${ }^{21}$ J. Snow, ${ }^{21}$ P.L. Wang, ${ }^{21}$ M. Wood, ${ }^{21}$ D.N. Brown, ${ }^{22}$ J. Fast,${ }^{22}$ R.L. McIlwain, ${ }^{22}$ T. Miao, ${ }^{22}$ D.H. Miller, ${ }^{22}$ M. Modesitt, ${ }^{22}$ D. Payne, ${ }^{22}$ E.I. Shibata, ${ }^{22}$ I.P.J. Shipsey, ${ }^{22}$ P.N. Wang, ${ }^{22}$ M. Battle ${ }^{23}$ J. Ernst, ${ }^{23}$ Y. Kwon, ${ }^{23}$ S. Roberts, ${ }^{23}$ E.H. Thorndike, ${ }^{23}$ and C.H. Wang ${ }^{23}$ (CLEO Collaboration)

${ }^{1}$ Southern Methodist University, Dallas, Texas 75275

${ }^{2}$ Syracuse University, Syracuse, New York 13244

${ }^{3}$ Vanderbilt University, Nashville, Tennessee 37235

${ }^{4}$ Virginia Polytechnic Institute and State University, Blacksburg, Virginia 24061

${ }^{5}$ Institute of Particle Physics, Carleton University, Ottawa, Ontario, Canada K1S $5 B 6$

${ }^{6}$ Institute of Particle Physics, McGill University, Montréal, Québec, Canada H3A 2T8

${ }^{7}$ California Institute of Technology, Pasadena, California 91125

${ }^{8}$ University of California, San Diego, La Jolla, California 92093

${ }^{9}$ University of California, Santa Barbara, California 93106

${ }^{10}$ Carnegie-Mellon University, Pittsburgh, Pennsylvania 15213

${ }^{11}$ University of Colorado, Boulder, Colorado 80309-0390

${ }^{12}$ Cornell University, Ithaca, New York 14853

${ }^{13}$ University of Florida, Gainesville, Florida 32611

${ }^{14}$ Harvard University, Cambridge, Massachusetts 02138

${ }^{15}$ University of Ilinois, Champaign-Urbana, Ilinois 61801

${ }^{16}$ Ithaca College, Ithaca, New York 14850

${ }^{17}$ University of Kansas, Lawrence, Kansas 66045

${ }^{18}$ University of Minnesota, Minneapolis, Minnesota 55455

${ }^{19}$ State University of New York at Albany, Albany, New York 12222

${ }^{20}$ Ohio State University, Columbus, Ohio, 43210

${ }^{21}$ University of Oklahoma, Norman, Oklahoma 73019

${ }^{22}$ Purdue University, West Lafayette, Indiana 47907

${ }^{23}$ University of Rochester, Rochester, New York 14627

(Received 11 March 1994)

\footnotetext{
*Permanent address: University of Hawaii at Manoa, Honolulu, HI 96822.

${ }^{\dagger}$ Permanent address: INP, Novosibirsk, Russia.
} 


\begin{abstract}
A measurement of the cross section for the combined two-photon production of charged pion and kaon pairs is performed using $1.2 \mathrm{fb}^{-1}$ of data collected by the CLEO II detector at the Cornell Electron Storage Ring. The cross section is measured at invariant masses of the two-photon system between 1.5 and $5.0 \mathrm{GeV} / c^{2}$, and at scattering angles more than $53^{\circ}$ away from the $\gamma \gamma$ collision axis in the $\gamma \gamma$ center-of-mass frame. The large background of leptonic events is suppressed by utilizing the CsI calorimeter in conjunction with the muon chamber system. The reported cross section is compared with leading order QCD models as well as previous experiments. Although the functional dependence of the measured cross section disagrees with leading order QCD at small values of the two-photon invariant mass, the data show qualitatively a transition to perturbative behavior at an invariant mass of approximately $2.5 \mathrm{GeV} / c^{2}$.
\end{abstract}

PACS number(s): 13.65.+i, 12.38.Qk, 14.40.Aq

\section{INTRODUCTION}

A measurement of the continuum production of meson pairs in two-photon reactions provides a test of quantum chromodynamics (QCD) for exclusive processes [1-5]. The leading order result based on perturbative QCD has been calculated by Brodsky and Lepage [1] for large center-of-mass scattering angle $\theta^{*}$ and large invariant mass $W$ of the two-photon system. Extensions of this work to finite values of $q^{2}$ (the invariant mass of the virtual photon) for one or both of the photons, as well as the incorporation of $\mathrm{SU}(3)$ flavor symmetry breaking effects are discussed by Gunion, Miller, and Sparks [2], and Benayoun and Chernyak [3], respectively. A calculation of the next-to-leading-order QCD prediction based on a particular model of the meson distribution amplitude is discussed by Nižić [4], while the running of $\alpha_{s}$ in the calculation of the meson form factor, and the subsequent effect on the leading order QCD result for $\gamma \gamma \rightarrow M^{+} M^{-}$ are discussed by Ji and Amiri [5].

The subject of this article is a measurement of the two-photon production of $\pi^{+} \pi^{-}$and $K^{+} K^{-}$using the CLEO II detector located at the Cornell Electron Storage Ring (CESR). The leading order result calculated by Brodsky and Lepage [1] can be expressed, approximately, in terms of the cross section for the two-photon production of muon pairs:

$$
\begin{aligned}
\frac{d \sigma}{d \cos \theta^{*}}\left(\gamma \gamma \rightarrow M^{+} M^{-}\right) \approx & \frac{4\left|F_{M}\left(W^{2}\right)\right|^{2}}{1-\cos ^{4} \theta^{*}} \\
& \times \frac{d \sigma}{d \cos \theta^{*}}\left(\gamma \gamma \rightarrow \mu^{+} \mu^{-}\right) .
\end{aligned}
$$

The angle $\theta^{*}$ denotes the scattering angle of one of the charged particles ("prongs") with respect to the $\gamma \gamma$ collision axis, calculated in the $\gamma \gamma$ center-of-mass frame. The overall angular dependence of Eq. (1) is $\sin ^{-4} \theta^{*}$ when one folds in the muon pair cross section. The form factor $F_{M}$ depends on the strong coupling constant $\alpha_{B}$, the $\gamma \gamma$ invariant mass $W$, and the pseudoscalar decay constant $f_{M}$. It is taken to be $\left(0.4 \mathrm{GeV}^{2} / c^{4}\right) / W^{2}$ for pions [1]. $A$ residual dependence on the pseudoscalar wave function outside of the form factor dependence is neglected in Eq. (1), and leads to a $\sim 25 \%$ negative correction. This model dependent correction is significantly larger for neutral mesons. Since the form factor is proportional to $f_{M}^{2}$, the ratio of the kaon pair cross section to the pion pair cross section depends on $\left(f_{K} / f_{\pi}\right)^{4}=2.2[6]$ when differences in the $\pi$ and $K$ wave functions are ignored. Benayoun and Chernyak [3] argue that such SU(3) flavor symmetry breaking effects are important and obtain a ratio closer to unity, but their absolute prediction for the combined pion pair plus kaon pair cross section does not differ appreciably from that of Brodsky and Lepage. The insensitivity of the combined cross section for charged pion and kaon pairs to the choice of wave function models makes this measurement an ideal probe of leading order perturbative QCD.

Both the Mark II and the TPC/Two-Gamma collaborations have published results $[7,8]$ on the high mass two-photon continuum production of charged pion and kaon pairs using data collected at the PEP $e^{+} e^{-}$storage ring at SLAC. Mark II data on the combined $\pi^{+} \pi^{-}$and $\mathrm{K}^{+} \mathrm{K}^{-}$cross section, measured in the dipion mass range between 1.7 and $3.5 \mathrm{GeV} / c^{2}$, suggest agreement with leading order QCD at values of $W_{\pi \pi}$ larger than $2 \mathrm{GeV} / c^{2}$ $\left(\left|\cos \theta^{*}\right|<0.5\right)$. TPC/Two-Gamma data on the $K^{+} K^{-}$ production cross section show reasonable agreement with the leading order QCD prediction in the measured $W$ range from 1.3 to $3.5 \mathrm{GeV} / c^{2}$ for $\left|\cos \theta^{*}\right|<0.6$. Charged pion pair data are about 3 times larger than the leading order QCD prediction for $W$ measured from 1.5 to $2.5 \mathrm{GeV} / c^{2}$ and $\left|\cos \theta^{*}\right|<0.3$, but at least some of the disagreement is likely to be caused by the interference of the $f_{2}(1270)$ resonance with the $\pi^{+} \pi^{-}$continuum. The leading order perturbative QCD calculations become more reliable at large $W$, so the experimental goal of a measurement of these two-photon processes is to probe larger values of $W$ than previously obtained, using higher statistics, to observe the transition in the cross section from nonperturbative to perturbative behavior.

We report on a measurement of the combined cross section for charged pion and kaon pairs [9] using the CLEO II detector at CESR. The measurement takes advantage of a much larger data sample than available at previous experiments, as well as a larger angular acceptance $\left(\left|\cos \theta^{*}\right|<0.6\right)$. The cross section is reported in the $W_{\pi \pi}$ range from 1.5 to $5.0 \mathrm{GeV} / c^{2}$ using $1.23 \mathrm{fb}^{-1}$ of integrated luminosity at a center-of-mass energy on or near the $\Upsilon(4 S)$ resonance $(\sqrt{s} \approx 10.6 \mathrm{GeV})$. A related study of the two-photon production of proton-antiproton pairs has recently been reported [10]. 


\section{EXPERIMENTAL SETUP}

\section{A. Detector}

The CLEO II detector [11] is a general purpose solenoidal detector designed to make precision measurements of both charged and neutral particles. Charged particle tracking is accomplished through the use of a 51 layer proportional drift chamber accompanied by a 10 layer intermediate wire chamber and a 6 layer straw tube chamber located just outside of the beryllium beam pipe. The transverse momentum resolution achieved within the $1.5 \mathrm{~T}$ field provided by the solenoidal magnet is given by $\left(\delta p_{\perp} / p_{\perp}\right)^{2}=\left(0.11 \% p_{\perp}\right)^{2}+(0.67 \%)^{2}$, with $p_{\perp}$ expressed in $\mathrm{GeV} / c$. The tracking system is surrounded by both a time-of-flight scintillation system, consisting of 64 counters arranged in $\phi$ (the azimuthal coordinate transverse to the beam axis) plus 28 counters per end cap, and an electromagnetic calorimeter composed of 7800 thallium-doped cesium iodide (CsI) crystals. The barrel section of the CsI calorimeter forms an integral part of this analysis as the fine tower segmentation allows for particle discrimination based on an analysis of shower shapes. The projective crystals are approximately $5 \times 5 \mathrm{~cm}^{2}$ at the front end and $30 \mathrm{~cm}$ long. The measured electromagnetic energy resolution is given by $\delta E / E=0.35 \% / E^{0.75}+1.9 \%-0.1 \% E$, with $E$ expressed in $\mathrm{GeV}$. All of these detector components lie within the 1.5 $\mathrm{T}$ superconducting solenoidal coil, which is in turn surrounded by 3 layers of iron interleaved with 3 sets of tracking chambers for muon detection. Each tracking chamber set contains three planar layers of Iarocci tubes with cathode strips providing information on the coordinate orthogonal to the wires. The barrel muon system is octagonal in cross section and the angular acceptance of the innermost superlayer is $|\cos \theta|<0.71(\theta$ is the polar angle measured with respect to the beam axis).

\section{B. Trigger}

The trigger is one of the more challenging aspects of an analysis of low multiplicity final states since it must meet the conflicting demands of high efficiency and large discrimination against beam-wall and beam-gas backgrounds. The components of the CLEO II detector used for this purpose include the tracking chambers, time-offlight scintillation counters, and calorimeter crystals-all of which are combined into a three level trigger system [12].

The two-prong trigger used in this analysis is designed to collect events containing either two minimum ionizing particles or at least one showering particle (electron or photon). The requirements of this trigger are the following: First, the time-of-flight system must register hits in at least two nonadjacent barrel scintillation counters. Second, the CsI calorimeter must identify either two separated energy clusters consistent with minimum ionizing particles ( $\sim 0.2 \mathrm{GeV}$ of deposited energy), or one energy cluster consistent with a showering particle $(>0.5 \mathrm{GeV}$ of deposited energy). Third, the tracking chambers must exhibit two clean track patterns such that the momentum transverse to the beam direction $\left(p_{\perp}\right)$ of one track is at least $0.4 \mathrm{GeV} / c$, while that of the other is at least $0.2 \mathrm{GeV} / c$. The exact trigger specification depends on the running period of the experiment, but at least one of the tracks must traverse the entire drift chamber.

Several methods were devised to measure the efficiency of the two-prong trigger from data. One technique makes use of events that satisfy a looser (prescaled) trigger to measure the efficiency of the additional trigger conditions in the standard two-prong trigger. Another technique exploits the redundancy of a calorimeter-only trigger to measure the efficiency of the tracking components of the two-prong trigger. Finally, a third technique uses fourprong events where two tracks are sufficient to fire the two-prong trigger. The trigger efficiency of the remaining two tracks is then studied. All three techniques are in very good agreement on the efficiency of those trigger components that are in common. The measured trigger efficiencies are listed separately for each trigger system in Table I. The measurenients refer to the average efficiency for two-prong events in the fiducial region $|\cos \theta|<0.7$ and with each track satisfying $p_{\perp}>0.5 \mathrm{GeV} / c$. When all trigger conditions are applied, the overall trigger efficiency for charged pion and kaon pairs is estimated to be $79 \%$ or $86 \%$ depending upon the running period of the experiment. A Monte Carlo simulation of the trigger system, calibrated to an independent data sample, confirms these values to within $5 \%$. The systematic uncertainty on the trigger efficiency is estimated to be $5 \%$.

The two-prong trigger runs at a rate of approximately $7 \mathrm{~Hz}$ at a luminosity of $2 \times 10^{32} \mathrm{~cm}^{-2} \mathrm{~s}^{-1}$. All of the events collected by the CLEO II trigger are passed through a software filter to remove obvious beam-gas and beam-wall backgrounds. This filter initially ran offline for the first $500 \mathrm{pb}^{-1}$ of data used in this analysis, but presently runs online as a part of the trigger system. It uses all of the tracking devices to reject events without clean track patterns originating from the nominal interaction point. Its efficiency can be measured directly from data since a prescaled sample of rejected events is saved for analysis, but the efficiency can also be determined by applying the filter to Monte Carlo events. For twophoton two-prong events, this efficiency is roughly $95 \%$.

\section{DATA ANALYSIS}

In this section we describe the analysis techniques used to extract the cross section for the two-photon production of charged pion and kaon pairs. The details of the

TABLE I. The measured efficiencies of various components of the two-prong trigger.

\begin{tabular}{lll}
\hline \hline System & & Efficiency (\%) \\
\hline Time-of-flight & & $98.0 \pm 1.0$ \\
Calorimeter & & $92.0 \pm 4.0$ \\
Tracking & early running & $88.0 \pm 2.0$ \\
& late running & $95.0 \pm 1.5$ \\
\hline \hline
\end{tabular}


event generation and detector simulation are discussed in Sec. III A. The event shape cuts used to select twophoton events from the data sample are described in Sec. III B. A technique for suppressing the large muon background in the two-photon event sample using information from the CsI calorimeter is presented in Sec. III C.

\section{A. Event simulation}

The extraction of the measured cross section for $\gamma \gamma \rightarrow$ $M^{+} M^{-}(M=\pi, K)$ relies on an accurate calculation of the QED process $e^{+} e^{-} \rightarrow e^{+} e^{-} \gamma^{*} \gamma^{*}$, where $\gamma^{*} \gamma^{*}$ are the intermediate virtual photons in the overall reaction. Rather than apply the equivalent photon approximation, which overestimates the $\gamma \gamma$ luminosity by about $15 \%$ in the $W$ range reported in this article, we rely on a Monte Carlo generator based on the formalism of Budnev et al. [13]. Only transverse photon polarization states are considered. The hadronic component of each nearly real photon is modeled by a $\rho$-pole form factor. The small but finite $q^{2}$ values of each photon cause these form factors to suppress the $\gamma \gamma$ luminosity by approximately $30 \%$ for the kinematic range explored by this experiment.

The event generation is completed by incorporating the Brodsky and Lepage [1] QCD model for $\gamma \gamma \rightarrow M^{+} M^{-}$; but for simplicity, the implicit $\sin ^{-4} \theta^{*}$ dependence of Eq. (1) is replaced with a flat $\cos \theta^{*}$ dependence. The total angular integral over $\cos \theta^{*}$ is scaled to the appropriate prediction of Eq. (1). The event generation is carried out separately for several ranges of $W$; each sample represents in excess of $2.9 \mathrm{fb}^{-1}$ of integrated luminosity.

The rest of the Monte Carlo simulation involves tracking the generated particles through a detector simulation package based on GEANT [14]. In an effort to simulate noise in the detector elements, random events selected by a dedicated trigger are embedded into the Monte Carlo sample. After a simulation of the trigger logic is performed, the events are then reconstructed in exactly the same manner as the data. The accuracy of most of the components of the event simulation is demonstrated in Sec. IV A by a comparison with two calculable QED processes.

\section{B. Event selection}

The $e^{+} e^{-} M^{+} M^{-}$final state system arises from the collision of quasi-real photons radiated by the electrons. Since the electrons scatter at very small angles, they generally go unmeasured in the experiment. Likewise, the $\gamma \gamma$ collision axis is nearly coincident with the electron beam axis, leaving the event $p_{\perp}$ unchanged. The energies of the radiated photons are not equal in general, so the final state system is Lorentz boosted with respect to the laboratory frame.

The event selection cuts exploit these general features of two-photon scattering at $e^{+} e^{-}$colliders to suppress the large background of annihilation events. Exactly two oppositely charged particles consistent with originating from the interaction point (within $5 \mathrm{~mm}$ transverse to the beam direction and $5 \mathrm{~cm}$ along it) are required to leave tracks in the drift chambers. The scalar sum of the two track momenta must be less than $8 \mathrm{GeV} / c$, and the total amount of energy deposited in the electromagnetic calorimeter must be less than $8 \mathrm{GeV}$ to suppress annihilation events (the beam energy is approximately $5.3 \mathrm{GeV}$ ). A total of $6.3 \times 10^{6}$ triggered events survive these preselection cuts.

As two-photon events tend to be well balanced in $p_{\perp}$, the total charged transverse momentum of the event is required to be less than $200 \mathrm{MeV} / c$. Annihilation produced tau events $\left(e^{+} e^{-} \rightarrow \tau^{+} \tau^{-}\right)$, one of the principal backgrounds to this measurement, tend not to satisfy this requirement owing to the missing momentum carried away by the neutrinos. (The expected level of tau pair contamination is addressed in Sec. IV B.) Similarly, the two tracks are required to be opposite in $\phi$ to within $50 \mathrm{mrad}$. To further ensure that there are no undetected particles other than the scattered electrons, the polar angle of the missing momentum vector of the event must point to within $10^{\circ}$ of the beam axis. Cosmic rays often trigger the experiment and may be classified as two-photon events if they pass near the interaction point. To reduce this background, as well as the contamination from annihilation events, the two tracks must pass an acolinearity requirement: the angle between the two tracks must be more than $100 \mathrm{mrad}$ away from back to back. Finally, the two tracks are required to project to well-understood regions of the detector and must fire a two-track trigger. The angular cut is $|\cos \theta|<0.7$ for each track. This directly restricts the angular acceptance in the $\gamma \gamma$ centerof-mass frame, and a cut is made to select events with $\left|\cos \theta^{*}\right|<0.6$ since there is little acceptance outside this range. With all event shape requirements applied, the data sample is reduced to $\mathbf{4 8 8 0 0 0}$ events.

Events containing electrons are suppressed by cutting on the quantity $E_{\mathrm{CC}} / p$. Since electrons will deposit most of their energy in the CsI calorimeter, the ratio of the energy deposit $E_{\mathrm{CC}}$ to the magnitude of the momentum $p$ measured in the tracking system will peak at 1.0. A cut is made to select only events with tracks satisfying $E_{\mathrm{CC}} / p<0.7$. Events with neutral energy observed in the detector are also rejected by requiring that the total amount of energy in the CsI calorimeter unassociated with tracks, $E_{\text {neut }}$, be less than $500 \mathrm{MeV}$. Both barrel and end cap calorimeter energy clusters are considered. Events with explicitly reconstructed $\pi^{0} s$ in them-identified as having two unmatched, isolated showers of at least $50 \mathrm{MeV}$ in energy that combine to within $15 \mathrm{MeV} / c^{2}$ of the nominal $\pi^{0}$ mass-are also rejected. This leaves 172000 events satisfying all cuts but the muon rejection criteria to be discussed in Sec. III C. Table II summarizes all cuts used in the two-prong selection.

Charged pions and kaons can be separated by $d E / d x$ or time-of-flight measurements. Unfortunately, the flight times for pions and kaons are the same to within experimental uncertainty for momenta greater than about $1.2 \mathrm{GeV} / c$ using the CLEO II time-of-flight system. Furthermore, the $d E / d x$ energy loss bands using the CLEO II drift chamber cross at about $1.0 \mathrm{GeV} / c$. Be- 
TABLE II. Event shape and acceptance cuts used in the two-prong selection.

\begin{tabular}{ll}
\hline \hline$N_{\text {track }}=2$ & (exactly two charged tracks) \\
$\sum_{\perp} Q_{\mathrm{i}}=0$ & (net charge of zero) \\
$d_{\perp}(i)<5 \mathrm{~mm}(i=1,2)$ & (impact parameter $\perp$ beam) \\
$d_{\|}(i)<5 \mathrm{~cm}(i=1,2)$ & (impact parameter $\|$ beam) \\
$\sum|p|<8 \mathrm{GeV} / c$ & (low total charged momentum) \\
$\sum E_{\mathrm{CC}}<8 \mathrm{GeV}$ & (low total calorimeter energy) \\
$p_{\perp}($ event $) \equiv\left|\mathbf{p}_{1 \perp}+\mathbf{p}_{2}\right|<200 \mathrm{MeV}$ & ( $\perp$ momentum balance) \\
Acoplanarity $\equiv|\pi-\Delta \phi|<0.05$ & (back-to-back in $\phi$ ) \\
Acolinearity $\equiv \mid \pi-$ arccos $\hat{p}_{1} \cdot \hat{p}_{2} \mid>0.1$ & (not back-to-back in 3D) \\
$\theta_{\text {miss }}>170^{\circ}$ or $<10^{\circ}$ & (missing momentum $\|$ beam) \\
$\left|\cos \theta_{i}\right|<0.7(i=1,2)$ & (tracks project to barrel) \\
$\left|\cos \theta^{*}\right|<0.6$ & (large angle scattering in \\
& the center-of-mass) \\
$E_{\mathrm{CC}} / p<0.7$ & (electron suppression) \\
$E_{\text {neut }}<500 \mathrm{MeV}$ & (low unmatched energy) \\
$\pi^{0}$ veto & (no reconstructible $\pi^{\circ} \mathrm{s}$ ) \\
Dedicated two-prong trigger & (trigger requirement) \\
Muon suppression & (see Sec. III C) \\
\hline \hline
\end{tabular}

cause of these limitations, pions and kaons will not be separated, and a combined cross section will be reported. Thus, the ratio of the $K^{+} K^{-}$to $\pi^{+} \pi^{-}$cross sections cannot be measured, and this forfeits one test of the $\gamma \gamma \rightarrow M^{+} M^{-}$models. The two-photon production of proton-antiproton pairs is negligible owing to the steep $W$ dependence of the cross section $[10,15]$.

\section{Muon rejection}

The primary challenge of the measurement of the twophoton production of charged pion and kaon pairs is to reduce the enormous two-photon background of muon pairs. Equation (1) predicts that the meson pair cross section will have an extra $W^{-4}$ dependence relative to the $\mu^{+} \mu^{-}$cross section, becoming several orders of magnitude smaller for $W$ of a few $\mathrm{GeV} / c^{2}$.

Muons can be identified by their penetration to a set of tracking chambers located outside several interaction lengths of material. However, a muon must have a momentum $p \gtrsim 1 \mathrm{GeV} / c$ in order to penetrate the first layer of iron and leave hits in the first set of tracking layers of the CLEO II muon chamber system. This restricts $W$ to be larger than approximately $2 \mathrm{GeV} / c^{2}$ for adequate muon rejection. In addition, the acceptance loss from the requirement that both tracks project sufficiently far from the cracks in the muon chamber system to limit the uncertainty due to multiple scattering is approximately $50 \%$.

An alternative method of separating muons from hadrons capitalizes on the different energy deposit patterns within the CsI calorimeter. It has the advantage of full azimuthal coverage (no cracks) and lower momentum reach, but it must be accomplished using the limited depth of the electromagnetic calorimeter. Muons typically leave a narrow trail of ionization and lose little energy as they traverse the $30-\mathrm{cm}$ depth of the calorimeter. Hadrons also usually deposit little energy since the electromagnetic calorimeter is only about an inter- action length deep. Occasionally, though, a pion or kaon will suffer an inelastic collision and deposit a significant amount of energy over a large lateral area. The energy deposited in the CsI calorimeter and the lateral shower size can be used to tag one of the mesons within two-prong events and thus suppress the muon pair background.

The lateral shower size is computed by calculating the root-mean-square (rms) width of the shower relative to the shower center-of-gravity. The center-of-gravity (cog) is computed by finding the energy-weighted mean of the locations of each crystal within all matched energy clusters, where the matching algorithm includes all showers within about $30 \mathrm{~cm}$ of the track projection. The angle $\theta_{\operatorname{cog}}$ is the polar angle of the shower center-of-gravity with respect to the beam axis. The shower width is calculated by taking the energy-weighted root-mean-square of the distance each crystal lies away from the center-ofgravity, which ought to be small for muons and noninteracting hadrons. The situation is complicated by the track curvature in the plane transverse to the beam direction, which makes the width depend on the transverse momentum. To separate this effect, the width is calculated in the $r-z$ plane only as Fig. 1 shows, where the $z$ axis is aligned with the beam direction and $r$ points from the interaction point to energy cluster. The rms width is calculated along just the $z$ axis, and is multiplied by $\sin \theta_{\operatorname{cog}}$ :

$$
\begin{aligned}
R_{\mathrm{z}} & =\sqrt{\frac{\sum_{i} E_{\mathrm{i}} d_{\mathrm{i}}^{2}}{\sum_{i} E_{\mathrm{i}}}} \\
d_{\mathrm{i}} & =\left(z_{\mathrm{i}}-z_{\mathrm{cog}}\right) \sin \theta_{\operatorname{cog}}
\end{aligned}
$$

The discrimination power of the shower energy and lateral size is evident in Figs. 2(a) and 2(b). These twodimensional plots show the total matched energy $E_{\mathbf{C C}}$ along the ordinate and the shower width $R_{\mathrm{z}}$ along the abscissa for particle momenta between 2 and $3 \mathrm{GeV} / c$. 


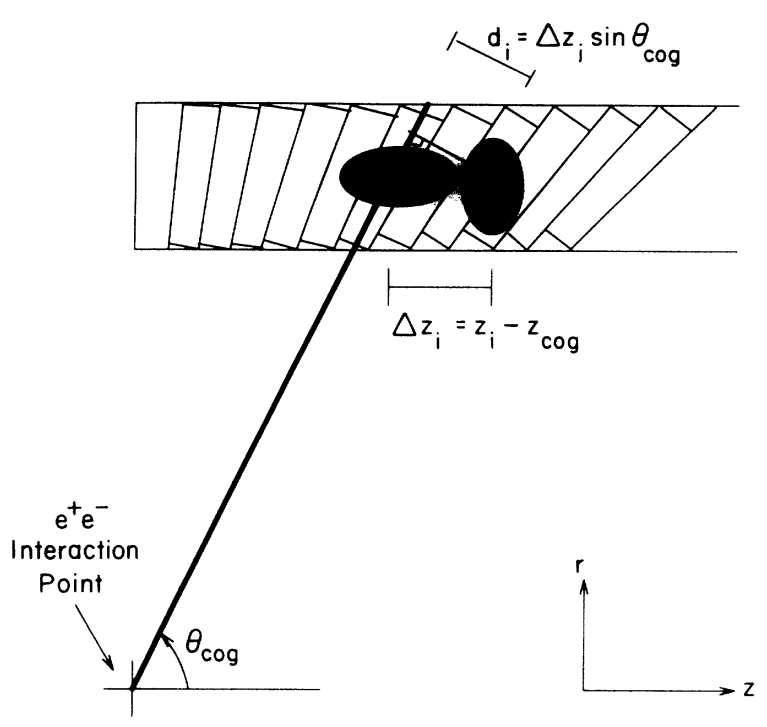

FIG. 1. Diagram illustrating the shower width calculation. The distance $d_{i}$ is taken to be the distance along $z$ from crystal $i$ to the shower center-of-gravity, multiplied by $\sin \theta_{\operatorname{cog}}$.

Tracks are restricted to the barrel of the detector $(|\cos \theta|<0.7)$. Muons selected from $e^{+} e^{-} \rightarrow \mu^{+} \mu^{-} \gamma$ are shown in 2(a), and charged pions from $\rho^{ \pm}$decays in two-prong annihilation produced tau events are shown in 2(b). A two-dimensional cut is very effective at separating muons from interacting charged hadrons. The lines shown in Fig. 2 define the acceptance region as $E_{\mathrm{CC}}>400 \mathrm{MeV}$ and $R_{z}>3.5 \mathrm{~cm}$. For momenta less than $3 \mathrm{GeV} / c$, only about 1 in 700 muons pass both cuts.

Figure 3 shows the efficiency for charged pions to lie in this acceptance region as a function of momentum $(|\cos \theta|<0.7)$. Data from $\rho^{ \pm}$decays are shown by the solid circles, and the efficiency is consistent with that determined from pions in $K_{s}^{0}$ decays as well as pions in four-prong events. A Monte Carlo simulation based on the detector simulation package (histogram) shows good agreement with the data. The charged kaon showering efficiency is expected to be similar to that of charged pions based on a Monte Carlo simulation.

The most efficient use of the shower selection criteria

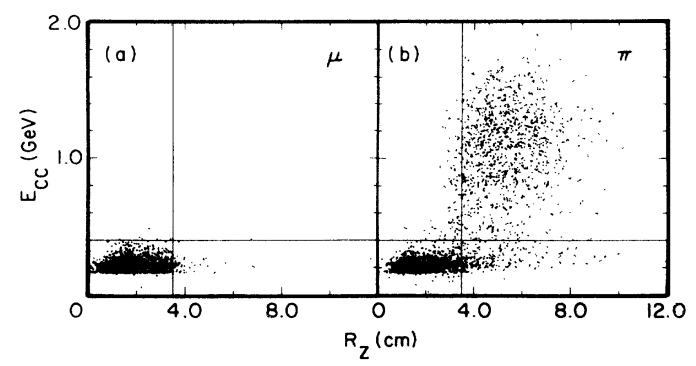

FIG. 2. Scatter plots of the matched CsI energy vs the shower width for muons (a) and pions (b). A particle is tagged as a hadron if $E_{\mathrm{CC}}>400 \mathrm{MeV}$ and $R_{z}>3.5 \mathrm{~cm}$.

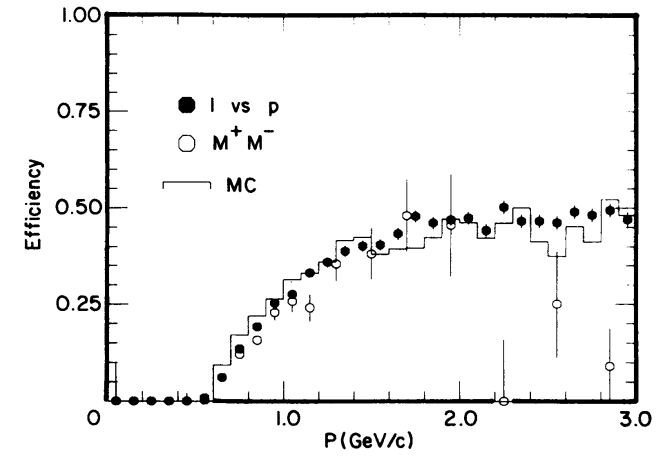

FIG. 3. The efficiency for charged pions and kaons to satisfy the shower tag as a function of momentum. Data shown include pions from $\rho^{ \pm}$decays as solid circles (1 vs $\rho$ ) as well as pions and kaons from two-prong events as open circles $\left(M^{+} M^{-}\right)$. A Monte Carlo simulation of the charged pion efficiency is shown by the histogram.

is to require just one track in a two-prong event to satisfy the shower shape cuts. This yields a $75 \%$ efficiency at large momenta, and a rejection factor for muon pair events of approximately 350. This allows a reach in $W$ of up to $5.1 \mathrm{GeV} / c^{2}$ before the two-photon muon production cross section equals that predicted for charged pion and kaon pairs combined. An advantage of following this procedure is that it provides a self-calibrating method of examining the purity of the data sample. One can tag one charged meson using the shower shape cuts and then measure the shower shape efficiency of the other particle. This method would also be sensitive to any deviation of the charged kaon efficiency from that of charged pions. The open circles in Fig. 3 represent this self-measured efficiency for two-prong events satisfying the two-photon event selection cuts summarized in Table II. The good agreement with the $\rho^{ \pm}$data for momenta less than $2 \mathrm{GeV} / c$ suggests that there is little muon contamination in that region. For momenta above $2 \mathrm{GeV} / c$, however, the self-measured efficiency is significantly lower than expected. The inferred contamination is well described by known background sources of muons (see Sec. IV B), and can easily be reduced to negligible levels using the muon chamber system since it is fully efficient at identifying muons (and thus capable of suppressing them) at these momenta. The tight geometric acceptance requirement to avoid the cracks in the muon system is not necessary to accomplish this final level of filtering. Therefore, to reject muons, we demand that at least one track satisfies the shower shape criteria, and that no muon chamber hits correlate to either track.

\section{RESULTS}

\section{A. Physics calibration}

The cross-section measurement of a reaction similar to the two-photon production of charged pion and kaon pairs, but theoretically well understood, checks our un- 
derstanding of the detector efficiencies as well as the modeling used at the generator level. Such a process is the two-photon production of muon pairs $\left(e^{+} e^{-} \rightarrow\right.$ $\left.e^{+} e^{-} \mu^{+} \mu^{-}\right)$. These events have a topology similar to $e^{+} e^{-} \rightarrow e^{+} e^{-} M^{+} M^{-}$, and the cross section for this process can be calculated to high precision within QED. However, the two-photon event shape cuts described in Sec. III B select not only two-photon events, but also radiative annihilation events. In particular, the process $e^{+} e^{-} \rightarrow \mu^{+} \mu^{-} \gamma$ cannot be distinguished from $e^{+} e^{-} \rightarrow$ $e^{+} e^{-} \mu^{+} \mu^{-}$when the radiated photon is at a small angle with respect to the beam axis-aside from the typically larger invariant mass of the two muons - since the $\mu^{+} \mu^{-}$ center-of-mass will be Lorentz boosted as in two-photon collisions.

All of the two-prong selection cuts discussed in Sec. III B are used to select the muon pair events. In addition, to obtain a clean sample of muons, both tracks are required to correlate to hits in the innermost superlayer of the muon chamber system. The invariant mass distribution of the selected events from $500 \mathrm{pb}^{-1}$ of data is shown in Fig. 4 (solid points). The spectrum does not begin until about $2 \mathrm{GeV} / c^{2}$ as the momentum of each particle must be large enough to penetrate the first layer of iron. The high $W$ cutoff is caused by the cut on the scalar sum of the track momenta. The histograms shown in Fig. 4 represent a Monte Carlo simulation of the reactions $e^{+} e^{-} \rightarrow e^{+} e^{-} \mu^{+} \mu^{-}$(dashed) and $e^{+} e^{-} \rightarrow \mu^{+} \mu^{-} \gamma(\gamma)$ (dotted). The sum of both contributions is represented by the solid histogram. The two-photon generator is the SIXDIA generator taken from Vermaseren [16]. The $e^{+} e^{-}$ annihilation generator is the FPAIR generator by Kleiss and van der Mark [17]. The two-photon events peak at low $W$, while the annihilation events peak at the machine center-of-mass energy and have a long radiative tail toward smaller values of $W$. A 3\% downward correction has been applied to the Monte Carlo trigger efficiency to account for differences with the efficiency measured from data. This correction was determined using processes other than $e^{+} e^{-} \rightarrow e^{+} e^{-} \mu^{+} \mu^{-}$(see Sec. II B) and carries

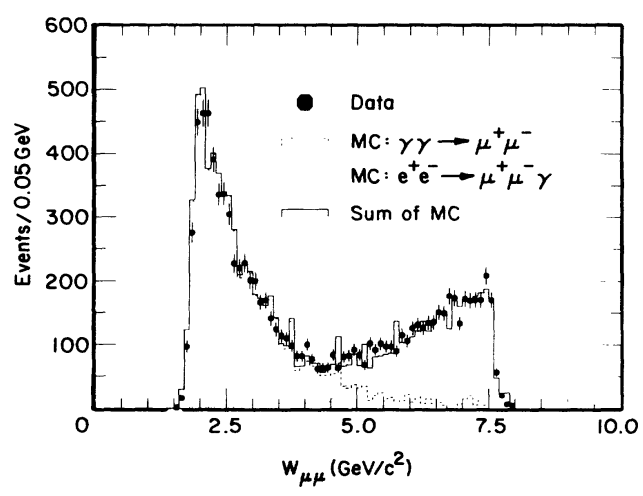

FIG. 4. Invariant mass distribution of muon pair events passing two-photon event selection criteria. The dashed histogram represents a simulation of $e^{+} e^{-} \rightarrow e^{+} e^{-} \mu^{+} \mu^{-}$, the dotted histogram a simulation of $e^{+} e^{-} \rightarrow \mu^{+} \mu^{-} \gamma$, and the solid histogram the sum of both. a $3 \%$ systematic uncertainty. The uncertainty and magnitude of this correction is smaller than that for charged pion and kaon pairs since the interaction of muons in the calorimeter is better understood.

As can be seen, the resulting simulation reproduces well the event yield across all of $W$. The areas of the two distributions agree to within $3 \%$, with the Monte Carlo slightly underestimating the event yield. This suggests that the modeling of the detector efficiencies (including the trigger efficiency) is well understood and that the theoretical calculations of Refs. [16] and [17] are in agreement with the data.

\section{B. Backgrounds}

Despite the rejection power of the event selection cuts described in Sec. IIIB, some background events still manage to contaminate the charged pion and kaon pair data sample. The two-photon background $e^{+} e^{-} \rightarrow$ $e^{+} e^{-} \mu^{+} \mu^{-}$is only expected to contribute about 1 event for $W>4 \mathrm{GeV} / c^{2}$ according to Monte Carlo calculations when one track is required to satisfy the shower tag described in Sec. III C. A larger contribution comes from the tail of radiative muon pair events $\left(e^{+} e^{-} \rightarrow \mu^{+} \mu^{-} \gamma\right)$. This source is expected to contribute $30 \pm 10$ events after the two-photon event shape and the shower tag requirements. The uncertainty in this estimate arises from the uncertainty in the muon rejection factor. This contamination was noticed in the shower tag efficiency extracted from the charged pion and kaon pair data sample itself (shown as open circles in Fig. 3), where $p>2 \mathrm{GeV} / c$ roughly corresponds to $W>4 \mathrm{GeV} / c^{2}$, but it is reduced to negligible levels when a cut is imposed to exclude any events with muon chamber hits correlating to either track.

Significant non-muon backgrounds arise from the tau pair production of charged mesons. In particular, the small sample of $e^{+} e^{-} \rightarrow \tau^{+} \tau^{-} \rightarrow \pi^{+} \pi^{-} \nu_{\tau} \bar{\nu}_{\tau}(\pi$ vs $\pi)$ events that pass the two-photon event shape cuts described in Sec. III B are problematic. From a Monte Carlo simulation using standard model parameters for the $\tau$ decays, $11 \pm 2 \pi$ vs $\pi$ events are expected to pass the shower tag on one of the tracks. An additional $4 \pm 3$ events are expected from the $K$ vs $\pi, K^{*}$ vs $\pi$, and $\rho$ vs $\pi$ channels. The $\mu$ vs $\pi$ background is negligible when tracks are required not to correlate to muon chamber hits. The combined $\tau$ background is distributed nearly uniformly across the invariant mass spectrum from about $1 \mathrm{GeV} / c^{2}$ to the effective cut at $8 \mathrm{GeV} / c^{2}$ (the invariant mass is calculated from the two charged final state particles assuming pion masses); therefore, we expect about $2 \tau$ events for each $1 \mathrm{GeV} / c^{2}$ wide bin in $W_{\pi \pi}$. This residual background becomes more than $5 \%$ of the total two-prong data sample for $W_{\pi \pi}>3 \mathrm{GeV} / c^{2}$, but it is subtracted from the two-prong data sample in the calculation of the two-photon charged pion and kaon pair cross section. 


\section{Charged pion and kaon pair cross section}

The extraction of the two-photon cross section from data involves comparing the two-prong event yields from data, $N_{\text {data }}$, and Monte Carlo, $N_{\mathrm{MC}}$, after all selection cuts have been imposed, and then scaling the average Monte Carlo $\gamma \gamma$ cross section appropriately:

$$
\begin{aligned}
\sigma_{\text {meas }} & \left(\gamma \gamma \rightarrow \pi^{+} \pi^{-}+K^{+} K^{-}\right) \\
= & \frac{N_{\text {data }}}{L_{\text {data }}}\left[\frac{L_{\mathrm{MC}}}{N_{\mathrm{MC}}} \bar{\sigma}_{\mathrm{MC}}\left(\gamma \gamma \rightarrow \pi^{+} \pi^{-}+K^{+} K^{-}\right)\right] .
\end{aligned}
$$

The term in brackets is $1 / \epsilon$, where $\epsilon$ is the overall efficiency as determined from the Monte Carlo, including geometrical acceptance and detection efficiencies. Specifically, in addition to the effects of topological cuts, loss of efficiency due to pions and kaons which decay in flight or punch through to the muon chambers is simulated in the Monte Carlo. A 5\% downward correction to the Monte Carlo trigger efficiency (in contrast to the $3 \%$ correction to the muon pair trigger efficiency) is determined from data. The factors $L_{\mathrm{MC}}$ and $L_{\text {data }}$ are the integrated luminosities of the Monte Carlo and data samples, respectively, and the number of events $N_{\text {data }}$ and $N_{\mathrm{MC}}$ are computed bin by bin in $\cos \theta^{*}$ and $W_{\pi \pi}$ (the twoprong invariant mass calculated assuming pion masses for the particles). The Monte Carlo cross section $\sigma_{\mathrm{MC}}$, integrated over the angular range $\left|\cos \theta^{*}\right|<0.6$, is given by

$$
\sigma_{\mathrm{MC}}\left(W_{\pi \pi}\right)=\frac{135 \mathrm{nb}}{W_{\pi \pi}^{6}}\left[1+2.2\left(\frac{W_{\pi \pi}}{W_{\mathrm{KK}}}\right)^{6}\right] .
$$

The cross section $\sigma_{\mathrm{MC}}$ is the combined cross section for the production of $\pi^{+} \pi^{-}$and $K^{+} K^{-}$. The factor 2.2 comes from $\left(f_{K} / f_{\pi}\right)^{4}$ in the QCD model of Brodsky and Lepage, and the factor $\left(W_{\pi \pi} / W_{K K}\right)^{6}$ comes from correcting $W$ to reflect kaon masses. The average value of this cross section for each bin in $\cos \theta^{*}$ and $W_{\pi \pi}$ is used in Eq. (3), but the dependence of $\sigma_{\text {meas }}$ on the choice of $\sigma_{\mathrm{MC}}$ is minimal since it cancels in Eq. (3) to first order. The measured cross section does depend on the calculation of the equivalent photon flux, however, in the determination of the factor $L_{\mathrm{MC}}$.

The data used in the extraction of the cross section are the two-photon two-prong candidates that pass the shower shape cut on at least one track and that exhibit no muon chamber tracks. A total of 6286 events satisfy these cuts. The final result, shown along with the QCD models of Brodsky and Lepage [1], and Benayoun and Chernyak [3], is presented in Fig. 5 as a function of $W_{\pi \pi}$. Only the statistical errors are shown. The yield in each $W_{\pi \pi}$ bin is given in Table III along with the detection efficiencies and measured cross sections. In the Brodsky and Lepage model the pseudoscalar wave function choice is arbitrary. Here we choose the perturbative wave function given in Ref. [1], which reduces the approximation given by Eq. (1) by about $25 \%$. The ratio of the $\gamma \gamma \rightarrow K^{+} K^{-}$cross section to the $\gamma \gamma \rightarrow \pi^{+} \pi^{-}$cross section in the Benayoun and Chernyak model is 1.08 rather than 2.20 as in the Brodsky and Lepage model, but Fig. 5 demonstrates that the sum of the two cross sections is nearly the same in both models.

The measured cross section agrees well in shape with the QCD models over the entire measured range in $W_{\pi \pi}$, though the normalization of the models is approximately $40 \%$ below the data. There is no compelling reason to expect that the cross section should exhibit good agreement at low $W_{\pi \pi}$, however, since perturbative calculations are not expected to be valid in this kinematic range and resonance formation should contribute. The last point plotted in Fig. 5 represents an upper limit at the $90 \%$ confidence level based on the observation of zero events (with a background expectation of two) in the $W_{\pi \pi}$ range from 4 to $5 \mathrm{GeV} / c^{2}$. When the veto on events containing reconstructed $\pi^{0} s$ is relaxed, two events do fall into this bin, but they are consistent with the tau process $e^{+} e^{-} \rightarrow \tau^{+} \tau^{-} \rightarrow \rho^{+} \pi^{-} \nu_{\tau} \bar{\nu}_{\tau}$, in agreement with the expected level of tau background.

The evolution of the $\cos \theta^{*}$ distribution as a function of the invariant mass $W$ can be used to test the $\sin ^{-4} \theta^{*}$ dependence of the QCD prediction. The Mark II measurement [7] did not have sufficient statistics to demonstrate such an angular distribution. We report a measurement of the differential pion and kaon pair cross section $d \sigma / d\left|\cos \theta^{*}\right|$ for four $W_{\pi \pi}$ bins, each containing three $\left|\cos \theta^{*}\right|$ bins. The result is shown in Figs. 6(a)-6(d). The angular bins are equally spaced in $\left|\cos \theta^{*}\right|$ from 0.0 to 0.6 , and the $W_{\pi \pi}$ ranges are $1.5-2.0,2.0-2.5,2.5-3.0$, and 3.0-4.0 GeV $/ c^{2}$. The leading order QCD prediction for $d \sigma / d\left|\cos \theta^{*}\right|$, taken from the Brodsky and Lepage model [1], is represented by the curves drawn in each figure. The measured cross section does exhibit a rise at small scattering angles similar to that expected by leading order perturbative QCD in the highest two $W_{\pi \pi}$ ranges. The behavior in the first range $\left(1.5<W_{\pi \pi}<2.0 \mathrm{GeV} / c^{2}\right)$ is opposite to that expected from QCD, while that in the second region $\left(2.0<W_{\pi \pi}<2.5 \mathrm{GeV} / c^{2}\right)$ is flat in $\cos \theta^{*}$. The disparity of the measured low $W_{\pi \pi}$ angular distribution with leading order QCD may be related to

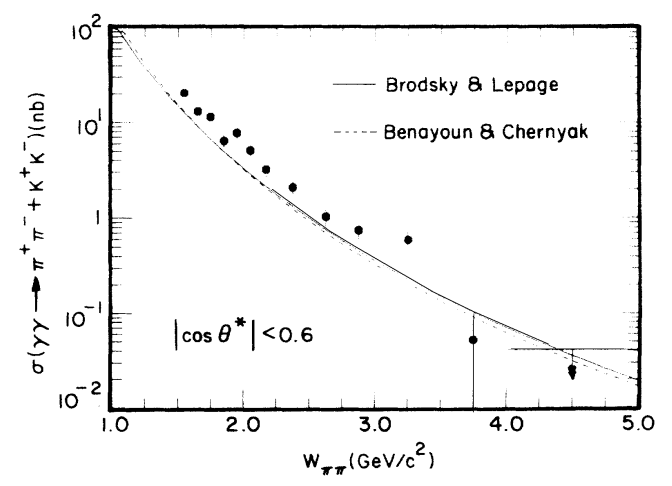

FIG. 5. Measured cross section for the two-photon production of charged pion and kaon pairs as a function of $W_{\pi \pi}$ in the angular region $\left|\cos \theta^{*}\right|<0.6$. Only statistical errors are shown. The leading order QCD predictions by Brodsky and Lepage, and Benayoun and Chernyak, are shown by the solid and dashed curves, respectively. 
TABLE III. The number of charged pion and kaon pair candidates passing all cuts for the $W_{\pi \pi}$ ranges shown in Fig. 5. Also shown is the detection efficiency as determined by Monte Carlo for $\left|\cos \theta^{*}\right|<0.6$, and the extracted cross section for the process $\gamma \gamma \rightarrow \pi^{+} \pi^{-}+K^{+} K^{-}$. Only statistical errors are shown.

\begin{tabular}{|c|c|c|c|}
\hline$\overline{W_{\pi \pi}\left(\mathrm{GeV} / c^{2}\right)}$ & Efficiency (\%) & Events & $\begin{array}{c}\sigma\left(\gamma \gamma \rightarrow \pi^{+} \pi^{-}+K^{+} K^{-}\right) \\
(\mathrm{nb})\end{array}$ \\
\hline $1.5-1.6$ & 2.6 & 245 & $20.5 \pm 1.8$ \\
\hline $1.6-1.7$ & 3.1 & 193 & $13.1 \pm 1.2$ \\
\hline $1.7-1.8$ & 3.6 & 161 & $11.5 \pm 1.2$ \\
\hline $1.8-1.9$ & 4.0 & 108 & $6.44 \pm 0.95$ \\
\hline $1.9-2.0$ & 4.4 & 117 & $7.79 \pm 1.08$ \\
\hline $2.0-2.1$ & 4.8 & 84 & $5.13 \pm 0.72$ \\
\hline $2.1-2.25$ & 5.4 & 54 & $3.18 \pm 0.53$ \\
\hline $2.25-2.5$ & 6.2 & 54 & $2.08 \pm 0.35$ \\
\hline $2.5-2.75$ & 7.3 & 23 & $1.03 \pm 0.23$ \\
\hline $2.75-3.0$ & 8.3 & 16 & $0.75 \pm 0.22$ \\
\hline $3.0-3.5$ & 9.9 & 21 & $0.59 \pm 0.15$ \\
\hline $3.5-4.0$ & 12.0 & 4 & $0.052 \pm 0.048$ \\
\hline $4.0-5.0$ & 15.0 & 0 & 0.041 (upper limit) \\
\hline
\end{tabular}

the nonperturbative nature of the cross section near the resonance region, and in particular, to the interference of the $f_{2}$ resonance with the $\pi^{+} \pi^{-}$continuum. Indeed, the angular dependence reported here is similar to the angular dependence of the charged pion pair cross section in the region near the $f_{2}$ (see, for example, Boyer et al. [18], and references therein).

The Mark II experiment performed a similar combined measurement of the charged pion and kaon pair cross section at the PEP $e^{+} e^{-}$storage ring [7]. A direct comparison of the measured cross sections is not straightforward, however, because of differing theoretical treatments used in the extraction of the two-photon cross section. The primary differences involve the use of the equivalent photon approximation in the Mark II analysis, and the appli-

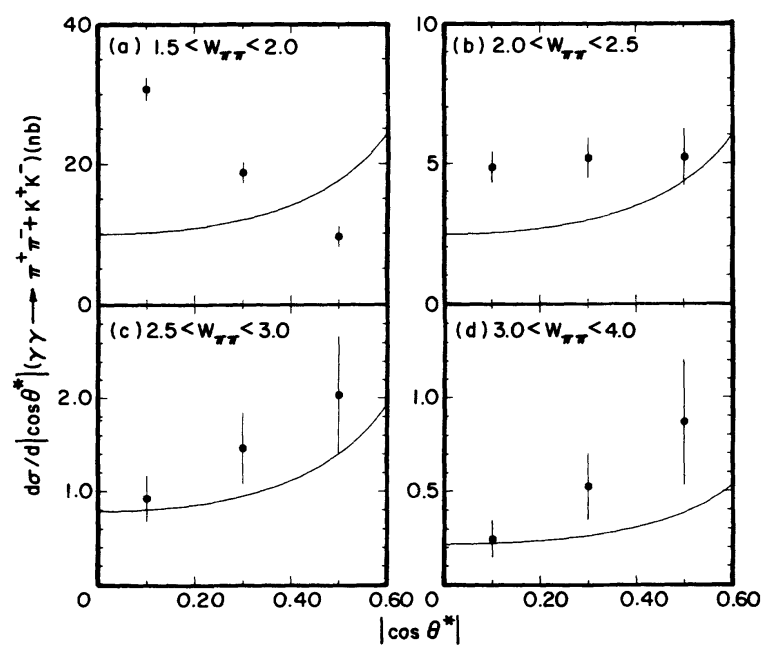

FIG. 6. The $\cos \theta^{*}$ dependence of the charged pion and kaon pair cross section for four bins in $W_{\pi \pi}$ : (a) 1.5-2.0 GeV/c $/ c^{2}$, (b) $2.0-2.5 \mathrm{GeV} / c^{2}$, (c) $2.5-3.0 \mathrm{GeV} / c^{2}$, and (d) 3.0-4.0 GeV $/ c^{2}$. The leading order QCD prediction by Brodsky and Lepage is represented by the solid curve. cation of a $\rho$ form factor to model the $q^{2}$ dependence of the virtual photons in our analysis. The combined effect of these two factors leads to an approximate $45 \%$ downward shift of the Mark II two-photon cross section relative to ours. Further differences include a smaller angular acceptance in the Mark II measurement $\left(\left|\cos \theta^{*}\right|<0.5\right)$ and a slightly different choice of $f_{K} / f_{\pi}$. When both analyses are treated in a similar manner, the measured cross sections of CLEO and Mark II agree to within $10 \%$.

The TPC/Two-Gamma Collaboration finds [8] that the $\pi^{+} \pi^{-}$cross section is about three times larger than the leading order QCD prediction (as given by the Brodsky and Lepage model) in the $W$ range from 1.5 to $2.5 \mathrm{GeV} / c^{2}$, and in the smaller angular region $\left|\cos \theta^{*}\right|<$ 0.3. Their kaon pair production cross section, though, is more in line with QCD in the measured $W$ range from 1.5 to $3.5 \mathrm{GeV} / c^{2}$, with $\left|\cos \theta^{*}\right|<0.6$. We find that in the narrow angular region $\left|\cos \theta^{*}\right|<0.3$, the TPC/TwoGamma data lie approximately $30 \%$ below our data on the combined cross section for pion and kaon pairs in the $W_{\pi \pi}$ range from 1.5 to $2.0 \mathrm{GeV} / c^{2}$. In this region our data are also several times larger than the leading order QCD prediction (see Fig. 6).

\section{Systematic effects}

Systematic uncertainties on the measured cross section involve uncertainties in the trigger efficiency $(\sim 5 \%)$ and the shower shape efficiency. The latter efficiency has been measured using various techniques (as shown in Fig. 3), all in good agreement with the detector simulation. The dependence of the measured cross section on the shower shape criteria has been studied by varying the cuts on the particle energy deposition in the calorimeter and the associated shower width by $25 \%$ of the nominal values. This range of values changes the overall event yield by as much as $50 \%$ for small $W_{\pi \pi}$, but leaves the extracted cross section unchanged to within $5 \%$. Moreover, the cross section has also been measured with the 
shower shape criteria applied to both detected particles rather than just one. Although the event yield is reduced approximately by two-thirds, the extracted cross section differs by less than $10 \%$. The shower shape criteria have also been applied to select charged pions in tau $\mu$ vs $\pi$ events, where several of the event shape cuts have been modified to select events with missing momentum rather than suppress them. The muon is identified by its penetration to the muon chamber system as in the calibration study of Sec. IV A. The resulting cuts are loose enough to select additional tau channels - notably channels containing neutral energy - but when all tau channels are included into the Monte Carlo generator, the resulting event yield agrees with that from data to $5 \%$. Finally, the two-photon cross section has been measured using only the muon chamber system in the limited kinematic region of adequate muon pair suppression. Results of this alternate approach confirm the functional dependence of our measurement of the cross section in both $W_{\pi \pi}$ and $\left|\cos \theta^{*}\right|$, but disagree on absolute normalization. Based on this study, and the previous tests of the shower shape efficiency, we assign a $20 \%$ systematic error to the overall normalization of our measurement, and a $10 \%$ systematic error on the point-to-point variation.

In addition to the modeling uncertainties described above, the measured cross section has a slight systematic dependence on the particular choice for the ratio of the kaon pair cross section to the pion pair cross section in the Monte Carlo generator through Eq. (3). This ratio is taken to be 2.2 , but if it is reduced to unity as suggested by Benayoun and Chernyak [3], the reported cross section will also be reduced. The reduction is largest at small $W_{\pi \pi}$, where the cross section is reduced by $12 \%$ for $W_{\pi \pi}<1.8 \mathrm{GeV} / c^{2}$. For $W_{\pi \pi}$ in the range from 1.8 to $2.75 \mathrm{GeV} / c^{2}$, the reduction is approximately $8 \%$, and for $W_{\pi \pi}$ larger than $2.75 \mathrm{GeV} / c^{2}$, the reduction is $3 \%$ or less. Finally, as noted before, removing the $\rho$ form factors in the modeling of the $q^{2}$ dependence of the virtual photons reduces our measured cross section by approximately $30 \%$.

\section{CONCLUSION}

We have presented data on the combined two-photon production of charged pion and kaon pairs from a sam- ple of $1.2 \mathrm{fb}^{-1}$ collected at CESR. Sufficient rejection of leptonic events is achieved through the novel use of the electromagnetic calorimeter as a tag on early interacting hadrons, which removes some of the fiducial and momentum constraints of the conventional muon chamber system. The $W_{\pi \pi}$ dependence of the measured cross section in the angular region $\left|\cos \theta^{*}\right|<0.6$ shows reasonable agreement with leading order perturbative QCD predictions across the entire measured range from 1.5 to $5.0 \mathrm{GeV} / c^{2}$, though the QCD-based models lie approximately $40 \%$ below the data. The $\cos \theta^{*}$ dependence of the cross section exhibits a rise at small scattering angles consistent with that expected by QCD for $W_{\pi \pi}>2.5 \mathrm{GeV} / c^{2}$. The magnitude of the measured cross section is in good agreement with results from the Mark II experiment, but is in disagreement by about $30 \%$ with results from the TPC/Two-Gamma experiment. Systematic uncertainties on the extracted cross section from detector acceptance effects amount to $20 \%$, while different theoretical treatments in the extraction of the cross section lead to variations of up to $30 \%$. The reported measurement extends the reach in $W$ further than previous experiments, allowing the observation of a transition in the cross section from nonperturbative to perturbative-like behavior.

\section{ACKNOWLEDGMENTS}

We gratefully acknowledge the effort of the CESR staff in providing us with excellent luminosity and running conditions. J.P.A. and P.S.D. thank the PYI program of the NSF, I.P.J.S. thanks the YI program of the NSF, G.E. thanks the Heisenberg Foundation, K.K.G., I.P.J.S., and T.S. thank the TNRLC, K.K.G., H.N.N., J.D.R., T.S. and H.Y. thank the OJI Program of the U.S. DOE and P.R. thanks the A.P. Sloan Foundation for support. This work was supported by the National Science Foundation and the U.S. Department of Energy.
[1] S. J. Brodsky and G. P. Lepage, Phys. Rev. D 24, 1808 (1981).

[2] J. F. Gunion, D. Miller, and K. Sparks, Phys. Rev. D 33, 689 (1986).

[3] M. Benayoun and V. L. Chernyak, Nucl. Phys. B329, 285 (1990).

[4] B. Nižić, Phys. Rev. D 35, 80 (1987).

[5] C.-R. Ji and F. Amiri, Phys. Rev. D 42, 3764 (1990).

[6] Particle Data Group, K. Hikasa et al., Phys. Rev. D 45,
S1 (1992)

[7] Mark II Collaboration, J. Boyer et al., Phys. Rev. Lett. 56, 207 (1986).

[8] TPC/Two-Gamma Collaboration, H. Aihara et al., Phys. Rev. Lett. 57, 404 (1986); W. G. J. Langeveld, Ph.D. thesis, State University, Utrecht, The Netherlands, 1985.

[9] More details can be found in D. Acosta, Ph.D. thesis, University of California, San Diego, 1993.

[10] M. Artuso et al., Cornell Report No. CLNS 93-1245 (un- 
published); B. Ong, Ph.D. thesis, University of California, San Diego, 1993.

[11] CLEO Collaboration, Y. Kubota et al., Nucl. Instrum. Methods Phys. Res., Sect. A 320, 66 (1992).

[12] CLEO Collaboration, C. Bebek et al., Nucl. Instrum. Methods Phys. Res., Sect. A 302, 261 (1991).

[13] V. M. Budnev et al., Phys. Rep. 15, 181 (1975).

[14] R. Brun et al., CERN Report No. DD/EE/84-1, 1987 (unpublished).
[15] G. R. Farrar, E. Maina, and F. Neri, Nucl. Phys. B259, 702 (1985); B263, 746(E) (1986); TPC/Two-Gamma Collaboration, H. Aihara et al., Phys. Rev. D 36, 3506 (1987).

[16] J. A. M. Vermaseren, Nucl. Phys. B229, 347 (1983).

[17] R. Kleiss and S. van der Marck, Nucl. Phys. B342, 61 (1990).

[18] Mark II Collaboration, J. Boyer et al., Phys. Rev. D 42, 1350 (1990). 


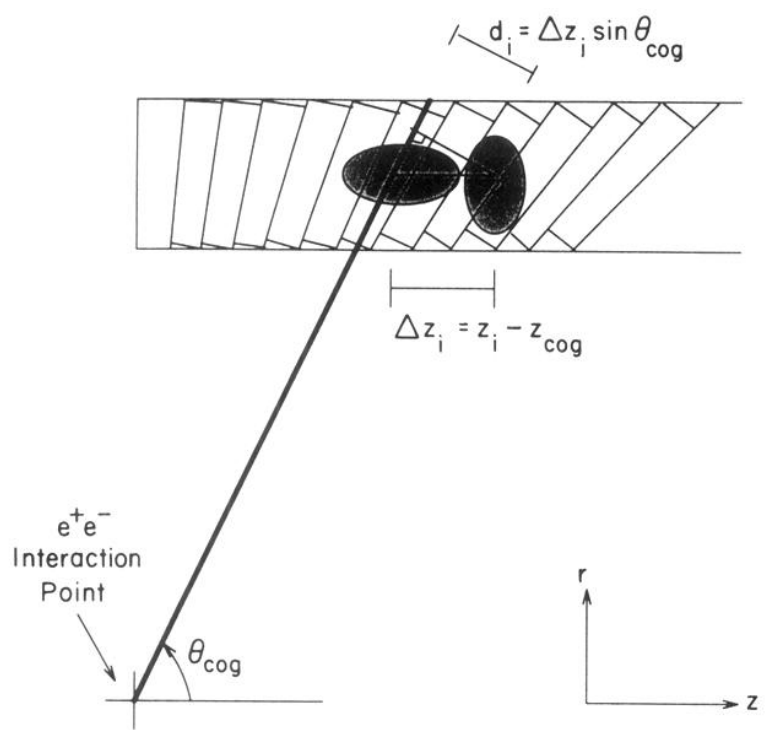

FIG. 1. Diagram illustrating the shower width calculation. The distance $d_{i}$ is taken to be the distance along $z$ from crystal $i$ to the shower center-of-gravity, multiplied by $\sin \theta_{\operatorname{cog}}$. 